\title{
Preon Model, Knot Algebra and Gravity
}

\author{
Risto Raitio \\ Espoo, Finland \\ Email: risto.raitio@gmail.com
}

How to cite this paper: Raitio, R. (2017) Preon Model, Knot Algebra and Gravity. Open Access Library Journal, 4: e3432. https://doi.org/10.4236/oalib.1103432

Received: February 6, 2017

Accepted: February 25, 2017

Published: February 28, 2017

Copyright (๑) 2017 by author and Open Access Library Inc.

This work is licensed under the Creative Commons Attribution International License (CC BY 4.0).

http://creativecommons.org/licenses/by/4.0/

\begin{abstract}
I study the properties of a preon model for the substructure of the standard model quarks and leptons. The goal is to establish both local and global group representations for the particles of the model. Knot theory algebra $S L q(2)$ is shown to be applicable to the model. Teleparallel gravity is discussed with an interesting result to hadronic physics. A tentative glimpse on quantum gravity is indicated.
\end{abstract}

\section{Subject Areas}

Particle Physics

\section{Keywords}

Preons, Standard Model, Knot Theory, Teleparallel Gravity, Quantum Gravity

\section{Introduction}

The purpose of this brief note is to study a spin $1 / 2$ preon model in order to give group theoretic structure to it. The model should fulfill three requirements: (i) suggest the basis for the standard model (SM) local gauge group structure $S U(3)$ $\times S U(2) \times U(1)$; (ii) provide a single global group structure for preons, quarks and lepton; and (iii) prepare a basis for introducing gravity into the model, with an applicable form of general relativity (GR). At first sight it seems difficult to achieve all the above goals, in particular, gravity has received until now very little attention in particle physics.

The preon model of this author [1] [2] has intuitive appeal since it suggests the gauge group structures $S U(2)$ and $S U(3)$ for the weak and strong interactions, respectively. Support for point (ii) above has been presented by Finkelstein [3] [4] using the global knot algebra $S L q(2)$. Thirdly, it has been long known that a Dirac field coupled to Einstein-Hilbert or other gravity may yield interesting results for both particles and spacetime [5]. In continuation to [5] I here discuss 
first a case in teleparallel gravity (TG) and then Weyl quantum gravity ${ }^{1}$. An interesting number is derived in the latter framework for the "quantum of matter", which is what is hoped for hadronic physics.

The organization of this note is the following. The preon model described in section 2. The group $S L q(2)$ is discussed in section 3. Teleparallel gravity is discussed in section 4 , and Weyl quantum gravity in section 5 . Sections 4 and 5 are of explorative nature. Finally, conclusion are made in section 6 .

\section{Preon Model}

Requiring charge quantization $\{0,1 / 3,2 / 3,1\}$ and fermionic permutation antisymmetry for same charge preons, I have defined four bound states of three light preons which form the first generation quarks and leptons [1] [2]

$$
\begin{aligned}
& u_{k}=\epsilon_{i j k} m_{i}^{+} m_{j}^{+} m^{0} \\
& \bar{d}_{k}=\epsilon_{i j k} m^{+} m_{i}^{0} m_{j}^{0} \\
& e=\epsilon_{i j k} m_{i}^{-} m_{j}^{-} m_{k}^{-} \\
& \bar{v}=\epsilon_{i j k} \bar{m}_{i}^{0} \bar{m}_{j}^{0} \bar{m}_{k}^{0}
\end{aligned}
$$

A useful feature in (1) with two same charge preons is that the construction provides a three-valued index for quark $S U(3)$ color, as it was originally discovered [6], the corresponding gauge bosons being in the adjoint representation. The weak $S U(2)$ left handed doublets can be read from the first two and last two lines in (1). The standard model gauge structure $S U(N), N=1,2$ is emergent in this sense from the present preon model. In the same way quark-lepton transitions between lines $1 \leftrightarrow 3$ and $2 \leftrightarrow 4$ in (1) are possible.

The preon and SM fermion group structure is better illuminated using the representations of the $S L q(2)$ group in the next Section 3.

The above gauge picture is supposed to hold in the present scheme up to the energy of about $10^{16} \mathrm{GeV}$. The electroweak interaction has the spontaneously broken symmetry phase below an energy of the order of $100 \mathrm{GeV}$ and symmetric phase above it. The electromagnetic and weak forces take separate ways at higher energies $\left(100 \mathrm{GeV} \ll E \ll 10^{16} \mathrm{GeV}\right.$ ), the latter restores its symmetry but melts away due to ionization of quarks and leptons into preons. The electromagnetic interaction, in turn, stays strong towards Planck scale, $M_{\mathrm{Pl}} \sim 1.22 \times 10^{19} \mathrm{GeV}$. Likewise, the quark color and leptoquark interactions suffer the same destiny as the weak force. One is left with the electromagnetic and gravitational forces only at Planck scale.

The proton, neutron, electron and $v$ can be constructed of 12 preons and 12 anti-preons. The construction (1) is matter-antimatter symmetric on preon level, which is desirable for early cosmology. The model makes it possible to create from vacuum a universe with only matter: combine e.g. six $m^{+}$, six $m^{0}$ and their antiparticles to make the basic $\beta$-decay particles. Corresponding antiparticles may occur equally well.

${ }^{1}$ A brief summary of cyclic conformal cosmology based on Higgs quartic coupling behavior and the Weyl tensor is given in [5]. 
The baryon number (B) is not conserved in this model: a proton may decay at Planck scale temperature by a preon rearrangement process into a positron and a pion. This is expected to be independent of the details of the preon interaction. Baryon number minus lepton number (B-L) is conserved.

I have at the moment no detailed form for a preon-preon interaction. Its details are not expected to be of primary importance. I suppose this attractive, non-confining interaction is strong enough to keep together the charged preons but weak enough to liberate the preons at high temperature or after very long time period. This interaction gives a small mass to quarks and leptons. On the other hand, it has been suggested [3] that preons may not appear as detector observable asymptotic free particles, i.e. have any independent degree of freedom, but are concentrations of energy-momentum at the crossings of a flux tube. This property would contradict the ionization of preon bound states assumed above.

One may now propose that, as far as there is an ultimate unified field theory within the standard model, it is a preon theory with only gravitational and electromagnetic interactions.

In the early universe, the strong and weak forces are generated only after massless preons combine into quarks and leptons at lower temperature. These two forces function only with short range within nuclei making atoms, molecules and chemistry possible. In a contracting phase of the universe the same processes take place in the reverse order.

\section{Knot Theory: Preons, Quarks and Leptons}

Early work on knots in physics goes back in time to 19th and 18th century [7] [8]. More recently Finkelstein has proposed a model based on the group $S L q(2)$ [3] [4]. The standard model field operators $\psi(x)$ are complemented in his model by knot factors $D$ as follows [9]

$$
\psi(x) \rightarrow \hat{\psi}(x) D_{m m^{\prime}}^{j}
$$

where $D_{m m^{\prime}}^{j}$ is a $2 j+1$ dimensional representation of the $S L q(2)$ algebra $(\hat{\psi}(x)$ also has the $\left(j, m, m^{\prime}\right)$ indices, see [4]).

The oriented 2-dimensional projection of a 3-dimensional knot can be assigned three coordinates $(N, w, r)$ where $N$ is the number of crossings, $w$ is the writhe and $r$ the rotation. One can transform to new coordinates $\left(j, m, m^{\prime}\right)$. These indices label the irreducible representations of $D_{m m^{\prime}}^{j}$ of the symmetry algebra of the knot, $S L q(2)$ by setting

$$
j=N / 2, m=w / 2, m^{\prime}=(r+o) / 2
$$

This linear transformations makes half-integer representations possible. The knot constraints require $w$ and $r$ to be of opposite parity, therefore $o$ is an odd integer. The knot $(N, w, r)$ may be labeled by $D_{w / 2,(r+o) / 2}^{N / 2}(a, b, c, d)$. Therefore, to the $(N, w, r)$ knot the following expression of the algebra is associated 


$$
D_{m m^{\prime}}^{j}(a, b, c, d)=\sum_{\substack{\left(n_{a}+n_{b}, n_{+}\right) \\ \delta\left(n_{c}+n_{d}, n_{-}\right)}} A_{m m^{\prime}}^{j}\left(q, n_{a}, n_{c}\right) \delta\left(n_{a}+n_{b}, n_{+}^{\prime}\right) a^{n_{a}} b^{n_{b}} c^{n_{c}} d^{n_{d}}
$$

where $\left(j, m, m^{\prime}\right)$ is given by (3), $n_{ \pm}=j \pm m, n_{ \pm}^{\prime}=j \pm m^{\prime}$ and $A_{m m^{\prime}}^{j}\left(q, n_{a}, n_{c}\right)$ is given by

$$
A_{m m^{\prime}}^{j}\left(q, n_{a}, n_{c}\right)=\left[\frac{\left\langle n_{+}^{\prime}\right\rangle_{1}\left\langle n_{-}^{\prime}\right\rangle_{1}}{\left\langle n_{+}\right\rangle_{1}\left\langle n_{-}\right\rangle_{1}}\right]^{1 / 2} \frac{\left\langle n_{+}\right\rangle_{1} !}{\left\langle n_{a}\right\rangle_{1} !\left\langle n_{b}\right\rangle_{1} !} \frac{\left\langle n_{-}\right\rangle_{1} !}{\left\langle n_{c}\right\rangle_{1} !\left\langle n_{d}\right\rangle_{1} !}
$$

where, $n_{-}=n_{c}+n_{d},\langle n\rangle_{q}=\frac{q^{n-1}}{q-1}$ and \langle\rangle$_{1}=\langle\rangle_{q_{1}}$.

One assigns physical meaning to the $D_{m m^{\prime}}^{j}$ in (4) by interpreting the a, b, c, and $d$ as creation operators for spin $1 / 2$ preons. These are the four elements of the fundamental $j=1 / 2$ representation $D_{m m^{\prime}}^{1 / 2}$ as indicated in Table 1 .

For notational clarity, I use in the Table 1 and Table 2. the preon names of [3]. The preon dictionary from the notation of [1] is the following:

$$
\begin{aligned}
m^{+} & \mapsto a, m^{0} \mapsto c \\
m^{-} & \mapsto d, \bar{m}^{0} \mapsto b
\end{aligned}
$$

The standard model particles are the following $D_{m m^{\prime}}^{3 / 2}$ representations "as indicated in Table 2.

All details of the $S L q(2)$ extended standard model are discussed in [9], including the gauge and Higgs bosons and a candidate for dark matter. I do not, however, see much advantage for introducing composite gauge bosons in the model (gauge invariance is a local property). Introduction of color is done slightly differently in [4]. In the early universe developments there is similarity between the knot and the present preon model. Therefore, apart from the differences in interpretation, the model of [1] and the knot algebra of [3] are equivalent in the fermion sector.

In summary, knots having odd number of crossings are fermions and knots

Table 1. The $D^{1 / 2}$ representation of the four preons.

\begin{tabular}{ccc}
\hline$m$ & $m^{\prime}$ & preon \\
\hline $1 / 2$ & $1 / 2$ & $\mathrm{a}$ \\
$1 / 2$ & $-1 / 2$ & $\mathrm{~b}$ \\
$-1 / 2$ & $1 / 2$ & $\mathrm{c}$ \\
$-1 / 2$ & $-1 / 2$ & $\mathrm{~d}$
\end{tabular}

Table 2. The $D^{3 / 2}$ representation of the standard model particles and their preon content.

\begin{tabular}{cccc}
\hline$m$ & $m^{\prime}$ & particle & preons \\
\hline $3 / 2$ & $3 / 2$ & electron & aaa \\
$3 / 2$ & $3 / 2$ & neutrino & $\mathrm{ccc}$ \\
$3 / 2$ & $-1 / 2$ & d-quark & abb \\
$-3 / 2$ & $-1 / 2$ & u-quark & cdd \\
\hline
\end{tabular}


with even number of crossings are correspondingly bosons. The leptons and quarks are the simplest quantum knots, the quantum trefoils with three crossings and $j=3 / 2$. At each crossing there is a preon. The free preons are twisted loops with one crossing and $j=1 / 2$. The $j=0$ states are simple loops with zero crossings.

\section{Teleparallel Gravity}

The previous Section 3 was largely about internal quantum numbers and their origin in $S L q(2)$. In this section I take a quick look 'outside' in spacetime and try to understand what kind of consequences gravity might offer for model building.

In [5] I paid attention to an extension of general relativity, the EinsteinCartan, or Einstein-Cartan-Kibble-Sciama (ECKS), theory of gravity. There curvature and torsion represented different degrees of freedom in spacetime. Curvature is caused by energy-momentum and torsion is due to spin. Another form of gravity is teleparallel gravity. It is equivalent to general relativity. It is a gauge theory of the Poincaré translation group with a force law. This gauge group is Abelian, like in electromagnetism. On parallelizable manifolds a vector field can be defined everywhere. Lie groups are parallelizable manifolds. The curvature is zero in TG, while the torsion is zero in GR. Torsion is an alternative to curvature and they are related to the same degrees of freedom of gravity. Curvature and torsion are, strictly speaking, properties of connections, not of spacetime.

General relativity is not unique, therefore theoretical reasons and Occam's razor will be used: an interesting result for a quantum of matter is derived [10] from teleparallel gravity, which originates from Einstein's attempt to unify gravity and electromagnetism [11]. For an introduction to TG see [12].

The geometrical basic concept of TG is the tangent bundle. In a general Riemannian spacetime $\boldsymbol{R}$, at each point $p$ with coordinates $x^{\mu}$, there is a Minkowski tangent space $M=T_{p} \boldsymbol{R}$, the fiber, on which the local gauge transformation of the $T_{x^{\mu}} \boldsymbol{R}$ coordinates $x^{a}$ takes place

$$
x^{\prime a}=x^{a}+\epsilon^{a}\left(x^{\mu}\right)
$$

where $\epsilon^{a}$ are the transformation parameters. As usual, $\mu$ is a spacetime index and $a$ a fiber frame index.

The dynamics of the theory is based on vierbeins (tetrads) $e_{\mu}^{a}$, not on the metric tensor $g_{\mu v}$. The relevant geometry is Weitzenböck geometry [13]. The Cartan connection has a key role

$$
\Gamma_{\mu \lambda v}=e_{\mu}^{a} \partial_{\lambda} e_{a v}
$$

The torsion associated with this connection is

$$
T_{\lambda v}^{\mu}=e_{a}^{\mu}\left(\partial_{\lambda} e_{v}^{a}-\partial_{\nu} e_{\lambda}^{a}\right)=e_{a}^{\mu} T_{\lambda v}^{a}
$$

where $T_{\lambda v}^{a}=\partial_{\lambda} e_{v}^{a}-\partial_{\nu} e_{\lambda}^{a}$.

The Christoffel symbols ${ }^{0} \Gamma_{\mu \nu \lambda}$ yield a zero torsion because of its symmetry properties. The Cartan connection and Christoffel symbols are related by the 
equation

$$
\Gamma_{\mu \lambda v}={ }^{0} \Gamma_{\mu \lambda \nu}+K_{\mu \lambda v}
$$

where

$$
K_{\mu \lambda v}=\frac{1}{2}\left(T_{\lambda \mu \nu}+T_{v \lambda \mu}+T_{\mu \lambda v}\right)
$$

is the contortion tensor. The Weitzenböck geometry, with vanishing curvature, and the Riemannian geometry, with vanishing torsion, are related by (10), from which follows

$$
e R(e) \equiv-e\left(\frac{1}{4} T^{a b c} T_{a b c}+\frac{1}{2} T^{a b c} T_{b a c}-T^{a} T_{a}\right)+2 \partial_{\mu}\left(e T^{\mu}\right)
$$

where $e=\operatorname{det}\left(e_{a}^{\mu}\right), R(e)$ is the scalar curvature and $T_{a}=T_{b a}^{b}$. Consequently, the Lagrangian density for TG is chosen as follows

$$
\mathcal{L}=-k e\left(\frac{1}{4} T^{a b c} T_{a b c}+\frac{1}{2} T^{a b c} T_{b a c}-T^{a} T_{a}\right)-\mathcal{L}_{M}
$$

where $k=1 / 16 \pi \quad(c=G=1)$ and $\mathcal{L}_{M}$ is the matter Lagrangian.

In (12) the geometrical part is the same as in Einstein-Hilbert gravity, so both have the same dynamical properties. In TG it is possible to define an energymomentum tensor. Equation (13) can be rewritten as follows

$$
\mathcal{L}=-k e \Sigma^{a b c} T_{a b c}-\mathcal{L}_{M}
$$

where

$$
\Sigma^{a b c}=\frac{1}{4}\left(T^{a b c}+T^{b a c}-T^{c a b}\right)+\frac{1}{2}\left(\eta^{a c} T^{b}-\eta^{a b} T^{c}\right)
$$

The field equations are derived from (14) by varying with respect to $e^{a \mu}$ and they are

$$
e_{a \lambda} e_{b \mu} \partial_{v}\left(e \Sigma^{b \lambda v}\right)-e\left(\Sigma_{a}^{b v} T_{b v \mu}-\frac{1}{4} e_{a \mu} T_{b c d} \Sigma^{b c d}\right)=\frac{1}{4 k} e T_{a \mu}
$$

This can be written in a more compact form

$$
\partial_{v}\left(e \Sigma^{a \lambda v}\right)=\frac{1}{4 k} e e_{\mu}^{a}\left(t^{\lambda \mu}+T^{\lambda \mu}\right)
$$

where $T^{\lambda \mu}=e_{a}^{\lambda} T^{a \mu}$ and

$$
t^{\lambda \mu}=k\left(4 \Sigma^{b c \lambda} T_{b c}^{\mu}-g^{\lambda \mu} \Sigma^{b c d} T_{b c d}\right)
$$

From the antisymmetry property $\Sigma^{a \mu \nu}=-\Sigma^{a v \mu}$ it follows

$$
\partial_{\lambda}\left[e e^{a}\left(t^{\lambda \mu}+T^{\lambda \mu}\right)\right]=0
$$

which is a local equilibrium equation. It gives rise to a continuity equation

$$
\frac{\mathrm{d}}{\mathrm{d} t} \int_{V} \mathrm{~d}^{3} x e e_{\mu}^{a}\left(t^{0 \mu}+T^{0 \mu}\right)=-\oint_{S} \mathrm{~d} S_{j}\left[e e_{\mu}^{a}\left(t^{j \mu}+T^{j \mu}\right)\right]
$$

Therefore, $t^{\lambda \mu}$ can be identified as the gravitational energy-momentum tensor. The total energy-momentum vector can be defined as 


$$
P^{a}=\int_{V} \mathrm{~d}^{3} x e e_{\mu}^{a}\left(t^{0 \mu}+T^{0 \mu}\right)
$$

where $V$ is the volume of the $3 \mathrm{D}$ space.

\section{Weyl Quantum Gravity}

In [10] the quantization of gravity is done in a stationary spacetime

$$
\mathrm{d} s^{2}=g_{00} \mathrm{~d} t^{2}+g_{11} \mathrm{~d} r^{2}+g_{22} \mathrm{~d} \theta^{2}+g_{33} \mathrm{~d} \phi^{2}
$$

The components of $g_{\mu v}$ are functions of $r$ and $\theta$ only. The value of $g_{00}<0$ gives the correct limit for Minkowski spacetime.

For (22) there are an infinite number of vierbeins obeying the relation $g_{\mu v}=e_{\mu}^{a} e_{a v}$. To avoid this problem the vierbein field is interpreted as a reference frame adapted by an observer in spacetime. Therefore this choice is made

$$
e_{\mu}^{a}=\left(\begin{array}{cccc}
\sqrt{-g_{00}} & 0 & 0 & 0 \\
0 & \sqrt{g_{11}} \sin \theta \cos \phi & \sqrt{g_{22}} \cos \theta \cos \phi & -\sqrt{g_{33}} \sin \phi \\
0 & \sqrt{g_{11}} \sin \theta \sin \phi & \sqrt{g_{22}} \cos \theta \sin \phi & \sqrt{g_{33}} \cos \phi \\
0 & \sqrt{g_{11}} \cos \theta & -\sqrt{g_{22}} \sin \theta & 0
\end{array}\right)
$$

adapted for a stationary observer. To obtain the gravitational energy one needs first the $\Sigma^{(0) 0 i}$ components which read (here (0) refers to $a=0$ )

$$
\begin{aligned}
& 4 e \Sigma^{(0) 01}=2\left(\sqrt{g_{33}}+\sqrt{g_{22}} \sin \theta\right)-\frac{1}{\sqrt{g_{11}}}\left[\sqrt{\frac{g_{33}}{g_{22}}}\left(\frac{\partial g_{22}}{\partial r}\right)+\sqrt{\frac{g_{22}}{g_{33}}}\left(\frac{\partial g_{33}}{\partial r}\right)\right] \\
& 4 e \Sigma^{(0) 02}=2 \sqrt{g_{11}} \cos \theta-\frac{1}{\sqrt{g_{22}}}\left[\sqrt{\frac{g_{11}}{g_{33}}}\left(\frac{\partial g_{33}}{\partial \theta}\right)+\sqrt{\frac{g_{33}}{g_{11}}}\left(\frac{\partial g_{11}}{\partial \theta}\right)\right] \\
& e \Sigma^{(0) 03}=0
\end{aligned}
$$

The authors restrict the attention to Schwarzschild spacetime for which $g_{00}=(1-2 M / r)=g_{11}^{-1}$ where $M$ is the black hole mass. Now the only non-zero $\Sigma^{(0) 0 i}$ component reads

$$
4 e \Sigma^{(0) 0 i}=4 r \sin \theta[1-\sqrt{(1-2 M / r)}]
$$

Recalling $E \equiv P^{(0)}$ one gets

$$
E=4 k \int \mathrm{d}^{3} x \partial_{i}\left(e \Sigma^{(0) 01}\right)=\int \mathrm{d}^{3} x \mathcal{H}
$$

Therefore $\mathcal{H}=4 k \partial_{i}\left(e \Sigma^{(0) 01}\right)$ which for Schwarzschild case yields

$$
\mathcal{H}=4 k \sin \theta\left[1-\frac{1-M / r}{\sqrt{1-2 M / r}}\right]
$$

which is the classical gravitational Hamiltonian density.

Weyl's prescription for quantizing a gravitational field is this

$$
\theta \mapsto \hat{\theta}, r \mapsto \hat{r}
$$

where $\hat{\theta}=i \alpha \partial_{r}, \hat{r}=r$ and $\alpha$ is a constant with dimension of distance. The commutator of these operators is 


$$
[\hat{\theta}, \hat{r}]=i \alpha
$$

from (9). The constant $\alpha$ is very small, $\alpha \ll 1$ because non-commutativity of $r$ and $\theta$ is not observed. Therefore $\sin \left(i \alpha \partial_{r}\right) \approx i \alpha \partial_{r}$. Thus the Hamiltonian $\mathcal{H} \mapsto \hat{\mathcal{H}}$ given by

$$
\hat{\mathcal{H}}=4 i k \alpha\left\{\left[1-\frac{1-M / r}{\sqrt{1-2 M / r}}\right] \partial_{r}+\frac{M / 2 r^{2}}{(1-2 M / r)^{3 / 2}}\right\}
$$

This operator is anti-hermitian and has therefore real eigenvalues from an equation of the form $\hat{\mathcal{H}} \psi=\epsilon \psi$ which is

$$
\partial_{r} \psi+g(r) \psi=0
$$

where

$$
g(r)=\left[1-\frac{1-M / r}{\sqrt{1-2 M / r}}\right]^{-1}\left[\frac{i \varepsilon}{4 k \alpha}+\frac{M / 2 r^{2}}{(1-2 M / r)^{3 / 2}}\right]
$$

The Hamiltonian density is dimensionless and therefore the eigenvalue $\epsilon=E / M$ is dimensionless with $E$ being the observable of the field. The solution of (31) is

$$
\psi=\psi_{0} \exp \left(-\int g(r) \mathrm{d} r\right)
$$

which becomes in the limit $M \ll r$

$$
\psi=\psi_{0} \exp \left(\frac{-i \epsilon r^{2}}{8 k \alpha M}\right)
$$

Finally, the boundary condition at the singular points $r=0$ and $r=2 M$, namely $\psi(0)=\psi(2 M)$, is required. For Schwarzschild spacetime $E=M$ which leads to $\epsilon=1$. The gravitational energy of TG is a classical observable, as is the eigenvalue of the above quantum equation. The calculation leads to the result

$$
M=n m_{0}
$$

where $n=1,2,3, \cdots, N$ is an integer, to give the right mass $M$, with $k=1 / 16 \pi$ and $m_{0}=\alpha / 4$. In SI units $m_{0}=\alpha c^{2} / 4 G$. This is the quantum of matter [10]. Numerically it is of the order of $0.1 \mathrm{GeV}$.

Finally, I mention a calculation supporting torsion in general together with SM quarks. In [14] the cosmological constant it is calculated using a fourfermion interaction in a massive Dirac field in a torsional model of gravity

$$
\mathcal{L}_{S}=3 / 2 \pi G e \hbar^{2}\left(\bar{\psi} \gamma^{i} \gamma^{5} \psi\right)\left(\bar{\psi} \gamma_{i} \gamma^{5} \psi\right)
$$

using this four-fermion interaction an estimate for the cosmological constant is obtained

$$
\Lambda=\frac{3}{16 M_{P l}^{4}}\left(\bar{\psi} \gamma_{i} \gamma^{5} \psi\right)\left(\bar{\psi} \gamma^{i} \gamma^{5} \psi\right)
$$

This $\Lambda$, induced by torsion, depends on spinor fields and is not constant in 
time. If the spinor fields can form a condensate the vacuum expectation value of $\Lambda$ behaves like a cosmological constant. Quark fields in quantum chromodynamics form a condensate with a vacuum expectation value $\langle 0|\bar{\psi} \psi| 0\rangle \approx$ $-(230 \mathrm{MeV})^{3}$. This energy scale is only about eight times larger than the observed $\Lambda$ value.

\section{Conclusions}

Spin $1 / 2$ and charge $\{0,1 / 3\}$ preon models have a sound group theoretical basis. It is hoped that the preon scheme [1] would provide a way towards a better understanding of the roles of all interactions. For that goal the weak and strong interactions are treated in this scenario unconventionally. They are emergent from the very basic fermion structure of the model (1). Gravity and electromagnetism are the 'original' interactions in big bang of cyclic cosmology.

Finkelstein proved the knotted preon model agrees with the Harari-Shupe (H-S) rishon model [15] [16]. As shown above, it also agrees with the present preon model $^{2}$, but the H-S model is quite different from the present model of section 2. For one, I do not think hypercolor is realistic for preon interactions.

On classical level there are alternatives to Einstein-Hilbert gravity like conformal, Einstein-Cartan and teleparallel gravity, the first two alternatives briefly discussed in [5]. The teleparallel theory has been considered as a gauge theory of the Poincaré translation group [12]. However, at short distances the generators of infinitesimal translations may not commute any more [17]. But notwithstanding, I gave an example of a teleparallel calculation, which ended up with an intriguing numerical value for a gravitational quantum of mass (35): $m_{0} \sim m_{\text {pion }}$. This is interesting for hadronic physics. I wish to return to this question later.

If there are spin $3 / 2$ three preon states a McDowell-Mansouri [18] type of gravity may be expected. A spin $3 / 2$ particle, beyond the $S M$, is predicted in any three preon model of spin $1 / 2$ preons.

More work is needed to clarify and gain consensus in the questions of gravity, quantum version with fermions in particular, and possible unification with electromagnetism.

\section{References}

[1] Raitio, R. (1980) A Model of Lepton and Quark Structure. Physica Scripta, 22, 197. https://doi.org/10.1088/0031-8949/22/3/002

[2] Raitio, R. (2016) Combinatorial Preon Model for Matter and Unification. Open Access Library Journal, 3, e3032.

[3] Finkelstein, R. (2017) On the SLq(2) Extension of the Standard Model and the Measure of Charge. International Journal of Modern Physics A, 32, Article ID:

\footnotetext{
${ }^{2} \mathrm{~A}$ historical remark: The present model was originally conceived at SLAC, during the week of the $\psi$ discovery in November 1974, assuming the c-quark to be a gravitationally excited u-quark of spin $1 / 2$ and charge $\{0,1 / 3\}$ heavy constituents. This idea was not favored by the local theorists, and later by others, and was not therefore developed to be published until much later. I have an early negligent preprint on structured quarks ( $q q \bar{q}$ ), HU-TFT 12-74 (1974). Harari's paper was received in April 1979, Shupe's in May 1979 by Physics Letters. My paper was received by Physica Scripta in January 1980 - after being first rejected by some other journals.
} 
1730001

[4] Finkelstein, R. (2014) SLq(2) Extension of the Standard Model. Physical Review D, 89, Article ID: 125020. https://doi.org/10.1103/PhysRevD.89.125020

[5] Raitio, R. (2017) On the Conformal Unity between Quantum Particles and General Relativity. Open Access Library Journal, 4, e3342. https://doi.org/10.4236/oalib.1103342

[6] Greenberg, O. (2009) The Color Charge Degree of Freedom in Particle Physics. In: Greenberger, D., Hentschel, K. and Weinert, F., Eds., Compendium of Quantum Physics, Springer-Verlag, Berlin Heidelberg, 109-111. https://doi.org/10.1007/978-3-540-70626-7_32

[7] Thomson, W. (1868) VI.-On Vortex Motion. Earth and Environmental Science Transactions of the Royal Society of Edinburgh, 25, 217-260. https://doi.org/10.1017/S0080456800028179

[8] Faddeev, L. and Niemi, A. (1997) Stable Knot-Like Structures in Classical Field Theory. Nature, 387, 58. https://doi.org/10.1038/387058a0

[9] Finkelstein, R. (2015) The SLq(2) Extension of the Standard Model. International Journal of Modern Physics A, 30, Article ID: 1530037. https://doi.org/10.1142/S0217751X15300379

[10] Ulhoa, S. and Amorim, R. (2014) On Teleparallel Quantum Gravity in Schwarzschild Space-Time. Advances in High Energy Physics, 2014, Article ID: 812691. https://doi.org/10.1155/2014/812691

[11] Einstein, A. (1930) Auf die Riemann-Metrik und den Fern-Parallelismus gegründete einheitliche Feldtheorie. Mathematische Annalen, 102, 685-697. https://doi.org/10.1007/BF01782370

[12] Aldrovandi, R. and Pereira, J. (2013) An Introduction to Teleparallel Gravity. Springer, New York. https://doi.org/10.1007/978-94-007-5143-9

[13] Cartan, E. (1980) In NATO ASIB Proceedings 58: Cosmology and Gravitation: Spin, Torsion, Rotation, and Supergravity, 489-491.

[14] Popławski, N. (2011) Cosmological Constant from Quarks and Torsion. Annalen der Physik, 523, 291-295. https://doi.org/10.1002/andp.201000162

[15] Harari, H. (1979) A Schematic Model of Quarks and Leptons. Physics Letters B, 86, 83-86. https://doi.org/10.1016/0370-2693(79)90626-9

[16] Shupe, M. (1979) A Composite Model of Leptons and Quarks. Physics Letters B, 86, 87-92. https://doi.org/10.1016/0370-2693(79)90627-0

[17] Townsend, P. (1977) Small-Scale Structure of Space-Time as the Origin of the Gravitational Constants. Physical Review D, 15, 2795.

[18] MacDowell, S. and Mansouri, F. (1977) Unified Geometric Theory of Gravity and Supergravity. Physical Review Letters, 38, 739. 
Submit or recommend next manuscript to OALib Journal and we will provide best service for you:

- Publication frequency: Monthly

- 9 subject areas of science, technology and medicine

- Fair and rigorous peer-review system

- Fast publication process

- Article promotion in various social networking sites (LinkedIn, Facebook, Twitter, etc.)

- Maximum dissemination of your research work

Submit Your Paper Online: Click Here to Submit

Or Contact service@oalib.com 